Pickering, Jeffrey 2002: Give me Shelter: Reexamining Military Intervention and the Monadic Democratic Peace, in: International Interactions 28: 4, 293-324.

Regan, Patrick M. 2000: Civil Wars and Foreign Powers: Outside Intervention in Intrastate Conflict, Ann Arbor, MI.

Regan, Patrick M. 2002: Third-Party Interventions and the Duration of Intrastate Conflicts, in: Journal of Conflict Resolution 46: 1, 55-73.

Rhodes, Edward 2003: The Imperial Logic of Bush's Liberal Agenda, in: Survival 45: 1, 131-154.

Rosenau, James N. 1969: Intervention as a Scientific Concept, in: Journal of Conflict Resolution 13: 2, 149-171.

Schreiber, Wolfgang (ed.) 2008: Das Kriegsgeschehen 2006. Daten und Tendenzen der Kriege und bewaffneten Konflikte, Wiesbaden.

Small, Melvin/Singer, J. David 1982: Resort to Arms: International and Civil Wars, 1816-1980, Beverly Hills.

Stern, Paul C./Druckman, Daniel 2000: Evaluating Interventions in History: The Case of International Conflict Resolution, in: International Studies Review 2: 1, 33-63.
Sullivan, Patricia L. 2008: At What Price Victory? The Effects of Uncertainty on Military Intervention Duration and Outcome, in: Conflict Management and Peace Science 25: 1, 49-66.

Tillema, Herbert K. 1989: Foreign Over Military Intervention in the Nuclear Age, in: Journal of Peace Research 26: 2, 179-196.

UCDP/PRIO 2009: Tajikistan, in http://www.pcr.uu.se/gpdatabase/gpcountry.php?id=152\&regionSelect=6-Central_and_ Southern_Asia\#, last access 09.08.2009.

Vasquez, John A. 1993: The War Puzzle, Cambridge.

Vlassenroot, Koen/Raeymakers, Timothy 2004: The Politics of Rebellion and Intervention in Ituri: The Emergence of a New Political Complex? In: African Affairs 103: 412, 385-412.

Walter, Barbara F. 2002: Committing to Peace: The Successful Settlement of Civil Wars, Princeton, NJ.

Wood, Elisabeth J. 2008: The Social Processes of Civil War: The Wartime Transformation of Social Networks, in: Annual Review of Political Science 11, 539-561.

Wheeler, Nicholas 2000: Saving Strangers. Humanitarian Intervention in International Society, Oxford.

Zangl, Bernhard/Zürn, Michael 2003: Frieden und Krieg. Sicherheit in der nationalen und postnationalen Konstellation, Frankfurt a. M.

\title{
Die neue US-Strategie für Afghanistan und Pakistan
}

\author{
Thomas Horlohe*
}

\begin{abstract}
The Obama Administration's new AfPak-Strategy implements the strategy change long asked for by security experts and the military. Striving to integrate military with non-military means, to engage neighbouring and regional powers, and emphasizing the development of Afghan security forces, AfPak offers a far better chance at progress in addressing the security challenges in and around 'Pashtunistan' than the non-strategy pursued by the Bush Administration. However, AfPak commits only limited additional military forces, sets very ambitious force-goals regarding the Afghan National Army and struggles to balance and integrate escalating military action with the civilian reconstruction effort. In particular AfPak offers little in terms of fighting corruption or providing alternatives to the poppy-economy. In escalating the anti-Taliban campaign in Pakistan, AfPak takes considerable political risks. Furthermore, AfPak seems to be undecided which strategic objective to give priority: 'The War on Terror' or sustained nation-building. Blending both and making the latter the prerequisite for the former has little appeal as an exit strategy.
\end{abstract}

Keywords: AfPak-Strategie, Aufstandsbekämpfung, Staatsaufbau, Krieg gegen den Terror AfPak-Strategy, counterinsurgency, nation-buildung, war against terror

\section{Entstehung der AfPak-Strategie}

Nach einer zweimonatigen Überprüfungsphase verkündete Präsident Barack Obama am Freitag, dem 27. März 2009 eine

\footnotetext{
* Dipl.-Pol. Thomas Horlohe ist Referatsleiter im Wissenschafts-, Wirtschaftsund Verkehrsministerium des Landes Schleswig-Holstein. Dieser Aufsatz wurde peer-reviewed. Er ist die überarbeitete und erweiterte Fassung eines Beitrags für die Sendereihe „Streitkräfte und Strategien“ des Norddeutschen Rundfunks.
}

neue Strategie der USA für Afghanistan und Pakistan. Die sogenannte AfPak-Strategie gibt vor, welche Ziele die westliche Führungsmacht künftig in dieser Konfliktzone mit welchen Mitteln verfolgen wird. Sie hat erhebliche Auswirkungen auf beide betroffenen Staaten, die Region, das NATO-Bündnis und auch auf die deutsche Sicherheitspolitik für Afghanistan. Dieser Aufsatz stellt die Entstehung der AfPak-Strategie, ihre Ziele, Instrumente und Merkmale dar, arbeitet die Veränderungen ge- 
genüber der bisherigen Sicherheitspolitik der Regierung Bush heraus, betrachtet die ersten Umsetzungsschritte ${ }^{1}$ und unterzieht die Strategie ${ }^{2}$ einer kritischen Beurteilung.

Bereits im Präsidentschaftswahlkampf bezeichnete der Kandidat Obama Afghanistan als „den Krieg, den wir gewinnen müssen“. ${ }^{3}$ Er legte sich darauf fest, möglichst schnell Truppen aus dem Irak abzuziehen, um die US-Streitkräfte in Afghanistan verstärken zu können. Am 10. Februar 2009 gab das Weiße Haus bekannt, dass die Überprüfung der US-Strategie für Afghanistan und Pakistan einer interministeriellen Arbeitsgruppe unter Leitung von Bruce Riedel übertragen werde. Riedel wurde für seine Aufgabe für 60 Tage von der Brookings Institution, der bedeutendsten liberalen Denkfabrik in den USA, freigestellt. Ihm wurden Richard Holbrooke, Sonderbotschafter für Afghanistan und Pakistan, und Michele Flournoy, Staatssekretärin für Verteidigungspolitik (Under Secretary of Defense for Policy), zur Seite gestellt.

Die Personalie Riedel war Programm und Präjudiz. Der frühere CIA-Antiterrorexperte hatte sich als scharfer Kritiker der Regierung Bush profiliert. Seit dem Frühjahr 2007 beriet er den Präsidentschaftskandidaten Obama. Im Mai 2007 geißelte er den Irak-Krieg als in jeder Hinsicht kostspieligen Irrweg, der nur der Strategie Al-Qaidas in die Hände spiele, die USA ,auszubluten“. 4 Mit der Ernennung Riedels zum Leiter der AfPak-Strategiestudie war klar, dass statt eines Ausstiegs aus dem Konflikt am Hindukusch eine Rückbesinnung auf den weltweiten Kampf gegen den islamistisch-fundamentalistischen Terrorismus angezeigt war.

Begonnen hatten die Vorarbeiten für die Strategieüberprüfung bereits unter der Regierung Bush. Riedels Arbeitsgruppe konnte daher auf eine Zwischenbilanz des Central Command (CENTCOM), des für den Irak sowie für Afghanistan und Pakistan zuständigen US-Regionalkommandos, zurückgreifen, ebenso auf eine Studie des Stabs des Nationalen Sicherheitsrats, auf eine Untersuchung des britischen ISAF-Regionalkommandos Süd in Afghanistan, auf eine Strategiestudie der NATO, Empfehlungen des US-Generalstabs und auf die Ergebnisse einer Fachkonferenz vom November 2008. ${ }^{5}$ Die interministerielle Arbeitsgruppe führte zahlreiche Gespräche mit Experten und regte Konsultationen mit Entscheidungsträgern an, wie das spektakuläre trilaterale Treffen von US-Außenministerin Clin- ton mit ihren afghanischen und pakistanischen Amtskollegen am 25. Februar in Washington. ${ }^{6}$

Parallel zur Arbeit der interministeriellen Arbeitsgruppe nahm eine öffentliche Debatte ihren Lauf. Mit Kommentaren und Namensartikeln in angesehenen US-Tageszeitungen und Stellungnahmen vor Parlamentsausschüssen versuchten Experten, die für unterschiedliche Perspektiven und politische Lager standen, die Erarbeitung der AfPak-Strategie (AfPak-Strategy Review) zu beeinflussen. Neben der obligatorischen staatsmännischen Wegweisung Henry Kissingers ${ }^{7}$ sind hier das Plädoyer Leslie Gelbs für einen strategischen Rückzug, ${ }^{8}$ die Analogien zur Truppenverstärkung im Irak (surge $)^{9}$ und zur „Vietnamisierung“10 sowie die pragmatischen Hinweise der RAND-Corporation zu nennen. ${ }^{11}$ Fast alle diese Ratschläge sind in der AfPak-Strategie wiederzuerkennen. Ihr Formulierungsprozess ist durch Partizipation, Pragmatismus und Konsens gekennzeichnet.

\section{Ziele, Instrumente und Merkmale}

\subsection{Umfassender Sicherheitsbegriff}

Der AfPak-Strategie liegt ein umfassender Sicherheitsbegriff zugrunde. Sicherheit wird nicht mehr nur militärisch definiert. Dies verdeutlichen die folgenden bildhaften Worte Obamas: „Der Feldzug gegen den Extremismus wird mit Kugeln und Bomben allein nicht erfolgreich sein. " 12 Das Verständnis der neuen US-Administration von Sicherheitspolitik hat sich dem europäischen, zumindest theoretisch und dem Anspruch nach, bemerkenswert weit angenähert. Bereits Bushs Außenminister Colin Powell und Condoleeza Rice hatten die grotesk ungleiche Mittelausstattung von Außen- und Verteidigungsministerium beklagt. Aber es war Robert M. Gates, der als Verteidigungsminister der Bush-Administration in seiner viel beachteten Rede vor der Kansas State Universität am 26. November 2007 nicht nur ein Umdenken, sondern ein Umsteuern gefordert hatte. Die US-Regierung habe nichtmilitärische Instrumente ihrer Außenund Sicherheitspolitik sträflich vernachlässigt. Zu häufig werde das Militär zur Erledigung ziviler Aufgaben zweckentfremdet.

6 Helene Cooper, „Obama capitalizes on world's good will“, International Herald Tribune, 27.2.2009. Wegen der ungelösten Grenzfrage zwischen beiden Ländern und zahlreicher Terrorangriffe in Afghanistan, die auf pakistanisches Territorium zurückverfolgt werden konnten und an denen pakistanische Geheimdienstkreise nachweislich beteiligt waren, gingen sich beide Außenminister bis dahin möglichst aus dem Wege. Hochrangige trilaterale Treffen hatte Riedel in seinem Foreign Affairs-Aufsatz vorgeschlagen, a.a.O. (Fn. 4), S. $35 \mathrm{f}$.

7 Henry A. Kissinger, „The way forward“, International Herald Tribune, 27.2.2009

8 Leslie H. Gelb, „How to leave“, International Herald Tribune, 14./15.3.2009.

9 Max Boot, Frederick Kagan und Kimberly Kagan, „How to surge“, ebd.

10 Arthur Keller, „Been there“, International Herald Tribune, 10.3.2009.

11 James Dobbins, Counterinsurgency in Afghanistan, Stellungnahme vor dem Streitkräfteausschuss des Senats am 26.2.2009; www.rand.org/pubs/testimonies/CT318/ und Seth G. Jones, „Going the Distance“, Washington Post, 15.2.2009.

12 The White House, Office of the Press Secretary (Hrsg.), Remarks by the President on a New Strategy for Afghanistan and Pakistan, Washington D.C. 27.3.2009. 
Was man militärisch gewonnen habe, werde anschließend beim zivilen Wiederaufbau verspielt..$^{13}$

Zu den fünf Zielen der neuen AfPak-Strategie zählen eine leistungsfähigere, verantwortungsvollere und fähigere Regierung in Afghanistan ebenso wie eine stabile und verfassungsmäßige Zivilregierung in Pakistan. Als wichtige Instrumente werden der zivilgesellschaftliche Wiederaufbau in Afghanistan, eine legitime Regierung und die Einbeziehung der Provinz- und Distriktsverwaltungen in den Aufbau der Zivilgesellschaft genannt. ${ }^{14}$

\subsection{Afghanistan und Pakistan als ein Kriegs- schauplatz}

AfPak: Schon der Name soll zum Ausdruck bringen, dass die neue US-Strategie auf einer neuen, umfassenden Sichtweise des Konflikts am Hindukusch beruht. Afghanistan und Pakistan werden gemeinsam betrachtet, als zwei Teilräume einer Konfliktzone, die nur durch eine Grenzlinie aus Kolonialzeiten künstlich getrennt sind, wie Holbrooke im Februar 2009 auf der Münchner Sicherheitskonferenz ausführte: „Wir bezeichnen das Problem häufig mit der Abkürzung ,AfPak'. Sie steht für ,Afghanistan-Pakistan'. Damit wollen wir nicht bloß acht Silben sparen. Es ist der Versuch zum Ausdruck zu bringen - und uns selbst immer wieder einzuprägen -, dass es sich um einen Kriegsschauplatz handelt, der sich auf beiden Seiten einer unglücklich festgelegten Grenze erstreckt, der Durand-Linie. Westlich der Grenze können NATO- und andere Truppen ungehindert operieren. Auf der Ostseite hat Pakistan territoriale Souveränität. Aber genau dort, östlich der Grenze, liegt das Problem des internationalen Terrorismus. “ 15

Sicherheitsexperten hatten schon lange gefordert anzuerkennen, dass das Problem nicht Afghanistan ist, sondern „Paschtunistan“, das Siedlungsgebiet der großen Volksgruppe der

13 Gates nahm kein Blatt vor den Mund: „Trotz Neueinstellungen gibt es nur etwa 6600 Beamte im Auswärtigen Dienst. Das ist weniger als die Besatzung einer einzigen Trägerkampfgruppe [unserer Marine]. Einer Schätzung zufolge stehen dieses Jahr 30 Prozent der Beamten in der Behörde für Wirtschaftliche Zusammenarbeit zur Pensionierung an, wertvolle Berufserfahrung, die wir nicht neu am Markt einkaufen können. [...]. Obwohl Public Relations in den Vereinigten Staaten erfunden wurde, sind wir erbärmlich schlecht darin dem Rest der Welt zu vermitteln, wofür wir als Gesellschaft und Kultur stehen: Freiheit, Demokratie, unsere Politik und unsere Ziele. Es ist ganz einfach peinlich, dass Al-Qaida besser darin ist, seine Botschaften über das Internet zu verbreiten als Amerika. Ein Diplomat eines anderen Landes fragte mich vor einigen Jahren: ,Wie hat es ein Mann in einer Höhle geschafft, besser zu kommunizieren als die größte Kommunikationsgesellschaft der Welt?' Tempo, Beweglichkeit und interkulturelle Kompetenz sind nicht unbedingt die Begriffe, die einem sofort einfallen, wenn man über die strategische Kommunikation der USA spricht “ Robert M. Gates, „Landon Lecture (Kansas State University), Manhatten, Kansas, 26.11.2007, www.defenselink.mil/speeches/ speech.aspx?speechid=1199: (Übersetzung, T.H.). Dem Vernehmen nach war diese Rede mitentscheidend dafür, dass Präsident Obama Gates bat, im Amt zu bleiben. Vgl. Elisabeth Bumiller, „Robert Gates: An American master of adaption“, International Herald Tribune, 31.3.2009. Die empirische Grundlegung dieses neuen Ansatzes lieferten Robert E. Hunter, Edward Gnehm und George Joulwan, Integrating Instruments of Power and Influence, Santa Monica (RAND) 2008.

14 The White House (Hrsg.), White Paper of the Interagency Policy Group's Report on U.S. Policy toward Afghanistan and Pakistan, o.O. (Washington, D.C.), o.J. (27.3.2009), hier: S. 1, 3f.,www.whitehouse.gov/assets/documents/Afghanistan_Pakistan_White-Paper.pdf.

15 Richard C. Holbrooke, Rede auf der 45, Münchner Sicherheitskonferen am 8.2.2009; www.security conference.de/konferenzen/rede.php?menu $2009=\$ m e n u \_k o n f e r e n z e n=\$ s p r a c h e=d e \& i d=266 \&$. Die Durand- Linie wurde 1893 für die Dauer von 100 Jahren als Grenze festgelegt und ist bis heute von Afghanistan völkerrechtlich nicht anerkannt worden.
Paschtunen, das sich auf beiden Seiten der Durand-Linie erstreckt. Die AfPak-Strategie macht nun dieses längst überfällige Zugeständnis an die Realität. Damit ist allerdings noch keine Lösungsperspektive entwickelt. Holbrooke, Riedel und Generalstabschef Michael Mullen lassen keinen Zweifel daran, dass auf beiden Seiten der Grenze unterschiedliche „Spielregeln“ gelten. Reguläre Truppen wollen die USA in Pakistan nicht einsetzen, weil die Regierung Pakistans dies nicht akzeptiert.

Mit der Erweiterung des Kriegsschauplatzes um Pakistan hat die Regierung Obama ihre Herausforderungen kurzerhand mehr als verdoppelt, ohne dass ihre Mittel mitgewachsen wären. Sie mutet sich und ihren Verbündeten sehr viel mehr zu als bisher. Konfliktanalytisch ist dieser Schritt konsequent. Militärstrategisch handelt es sich um eine horizontale Eskalation, deren Folgen noch nicht zu übersehen sind. Unter den vielen Hintergrundinformationen, die über den Formulierungsprozess der AfPak-Strategie in die Öffentlichkeit drangen, weist keine darauf hin, dass zuvor eine Folgenabschätzung versucht wurde. ${ }^{16}$

\subsection{Internationalisierung und Regionalisierung}

Die Nachbarstaaten und die internationale Staatengemeinschaft sollen in die Lösung des Konflikts in der Region aktiv eingebunden werden. „Regionalisierung und Internationalisierung “ist eines von fünf Zielen der AfPak-Strategie. Den Vereinten Nationen (VN) ist dabei eine „wichtige Führungsrolle“ zugedacht. Die NATO-Verbündeten, Australien, Japan, Saudi Arabien, die Vereinigten Arabischen Emirate, der Iran, die Türkei, China und Russland sollen in unterschiedlichen Rollen unterstützen. Japan finanziert für das nächste halbe Jahr die Gehälter der afghanischen Polizei. ${ }^{17}$ Die NATO-Verbündeten sollen, wie überwiegend bereits zugesagt, mindestens bis zur Präsidentschaftswahl im August 2009 ihre Truppenkontingente aufstocken. Von Frankreich, Italien, den Niederlanden, Portugal, Rumänien und Spanien erwartet man Ausbildungshilfe für die afghanische Polizei. ${ }^{18}$ Saudi Arabien und die Vereinigten Arabischen Emirate sollen auf Pakistan Einfluss nehmen, Finanzhilfen beisteuern und zwischen der Regierung Karsai und einigen Talibangruppen und Milizen vermitteln. Der saudische Außenminister und der Chef des saudischen Geheimdienstes sind in dieser Rolle bereits seit einiger Zeit aktiv. ${ }^{19}$ Indien, der Erzfeind Pakistans, soll sich im Kaschmir-Konflikt zurückhalten und auf Angriffe terroristischer Gruppen besonnen reagieren, damit die pakistanische Armee sich stärker auf die Bekämpfung

16 Die Erfahrungen der USA mit horizontaler Eskalation nicht-konventioneller Kriege sind jedenfalls nicht ermutigend. „Erinnern Sie sich an den Einmarsch nach Kambodscha, um Vietnam zu retten?“, fragt der ehemalige CIA-Resident in Kabul, Graham Fuller, „Out of Afghanistan“, International Herald Tribune, 9.-10.5.2009.

17 Holbrooke, Press Briefing, a.a.O. (Fn. 5), S. 7.

18 Vgl. Thom Shanker und Steven Erlanger, „Afghanistan presents a choice of fusion or fracture“, International Herald Tribune, 03.4.2009.

19 Vgl. Carlotta Gall, „Kabul feels out Taliban on peace talks“, International Herald Tribune, 31.10.2008.; John F. Burns und Abdul Waheed Wafa, „Afghan leader asks Saudis to broker Taliban talks“, International Herald Tribune, 1.10.2008, und „Taliban offenbar zu Gesprächen mit Karzai bereit“, Frankfurter Allgemeine Zeitung, 17.3.2009, unter Berufung auf die Sunday Times. 
der Taliban konzentriert. ${ }^{20}$ Die von Indien in Westafghanistan fertig gestellte Fernstraße ermöglicht es der NATO, die Verbindung zum iranischen Hafen Chah Bahar in ihre Überlegungen zu alternativen Nachschubwegen einzubeziehen, nachdem sich die Anschläge auf Depots in Pakistan und auf die Versorgungsroute über den Khaiberpass häufen. ${ }^{21}$ Die Kooperationsbereitschaft Irans ist erforderlich, um die Grenze zur pakistanischen Provinz Belutschistan zu sichern, den Drogenschmuggel zu bekämpfen und die Flüchtlingsprobleme zu bewältigen. Das Treffen Holbrookes mit dem stellvertretenden iranischen Außenminister Mehdi Achundsadeh auf der Afghanistan-Konferenz am 31. März 2009 in Den Haag wurde als erster spektakulärer Erfolg der Regionalisierungspolitik gewertet. ${ }^{22}$

Das Beispiel Russlands zeigt, dass die USA für die Unterstützung ihrer AfPak-Strategie unter Umständen auch einen Preis bezahlen müssen. Auf Drängen Russlands drohte Kirgistan den Pachtvertrag der US-Luftwaffe für den Stützpunkt Manas auslaufen zu lassen, über den die US-Streitkräfte in Afghanistan versorgt werden. Gleichzeitig erklärte Russlands Außenminister Sergej Lawrow die Bereitschaft seines Landes, mit der NATO bei der Lösung ihrer Nachschubprobleme besser zusammenzuarbeiten. ${ }^{23}$

Die erklärte Einsicht, dass die AfPak-Strategie der Unterstützung anderer Staaten bedarf, ist überfällig und ein Fortschritt gegenüber der Regierung Bush. Die Interessen der Nachbarländer und Regionalmächte in Afghanistan sind allerdings sehr unterschiedlich und stimmen nur teilweise mit denen der USA überein. Der Regionalisierungsansatz kann einen Beitrag zur Konfliktregulierung leisten. Er sollte indessen nicht überschätzt werden.

\subsection{Verstärkung der US-Truppen}

Schon am 17. Februar 2009 ordnete Obama als erstes, frühes Zwischenergebnis des noch laufenden AfPak-Strategy Review knapp und unspektakulär an, die US-Truppen in Afghanistan um 17.000 Mann Kampftruppen und Kampfunterstützungstruppen aufzustocken. ${ }^{24}$ Mit der Bekanntgabe der AfPak-Strategie entschied Präsident Obama, weitere 4.000 Ausbilder zu-

20 „Einer der Gründe, warum der regionale Ansatz so wichtig ist, ist die Entspannung an der Grenze zu Kaschmir. Auf diese Weise wäre das pakistanische Militär nicht vollständig an dieser Grenze gebunden und in der Lage, sich hinsichtlich Ausbildung und Ausrüstung auf die Aufstandsbekämpfung an der Westgrenze zu konzentrieren." US-Generalstabschef Michael Mullen am 27.3.2009, (Übers. T.H.); www.jcs.mil/chairman/speeches/ 0327officepressavail\%20.pdf.

21 Thom Shanker und Elisabeth Bumiller, „U.S. Weighs NATO-Iran route to fight Taliban“, International Herald Tribune, 12.3.2009.

22 „Holbrooke trifft iranischen Regierungsvertreter“, Frankfurter Allgemeine Zeitung, 1.4.2009.

23 Ellen Barry und Michael Schwirtz, „U.S. scrambles to save base in Kyrgystan“, International Herald Tribune, 5.2.2009; „,Russia open to better cooperation with NATO“, International Herald Tribune, 12.2.2009; Clifford J. Levy, „Russia of two minds on Afghanistan“, International Herald Tribune, 23.2.2009.

24 Davon 8.000 Mann Marineinfanterie aus Camp Lejeune noch im Frühjahr 4.000 Mann Heereskampftruppen und 5.000 Mann Kampfunterstützungstruppen aus Fort Lewis bis zum Sommer. Vgl. Helene Cooper, „U.S. adds 17,000 troops to Afghan war“, International Herald Tribune, 19.2.2009. Ausschlaggebend dürfte die Absicht gewesen sein, die Präsidentschaftswahl in Afghanistan im August abzusichern. Wegen der langen Vorlaufzeiten für Truppenverlegungen war eine schnelle Entscheidung erforderlich, mit Rückwirkungen auf den Zeitplan für den Truppenabzug aus dem Irak, über den Obama zehn Tage später abschließend entschied. Vgl. Peter Baker, „U.S. Combat forces to quit Irak in 2010“, International Herald Tribune, 28.2./1.3.2009. sätzlich zu entsenden, um den Aufbau der afghanischen Armee voranzutreiben. Damit waren alle Forderungen des damaligen US-Befehlshabers in Afghanistan für 2009 erfüllt. Forderungen nach zusätzlichen 30.000 Soldaten, die zwischenzeitlich in die Diskussion gebracht worden waren, ${ }^{25}$ gab der Präsident nicht nach. Er gestand der Generalität genau das zu, was sie zuletzt von seinem Vorgänger verlangt hatte, nicht mehr. ${ }^{26}$

Eine massive oder dramatische Truppenverstärkung ist das nicht. Legt man den Maßstab der einschlägigen Dienstvorschrift von Heer und Marinekorps zugrunde, bedürfte es für Afghanistan 271.652 Soldaten, um die Aufständischen erfolgreich bekämpfen zu können. ${ }^{27}$ Kritische Beobachter der militärischen Entwicklung in Afghanistan halten die Verstärkung denn auch nicht für ausreichend. ${ }^{28}$

\subsection{Aufbau der afghanischen Sicherheitskräfte}

Die afghanische Armee soll von derzeit etwa 82.000 Mann bis 2011 auf eine Stärke von 134.000 gebracht werden, die Polizeikräfte im gleichen Zeitraum von 80.000 auf 82.000. Auch diese Zielwerte stellen keine Anhebung gegenüber denen dar, die bereits im August 2008 unter der Regierung Bush festgelegt worden waren. ${ }^{29}$

Berücksichtigt man die ernüchternden Erfahrungen beim Aufbau der Afghanischen Nationalarmee (ANA), ${ }^{30}$ so erscheint das quantitative Ziel von 52.000 zusätzlichen Soldaten in knapp drei Jahren und das qualitative Ziel „selbständiger“ Streitkräfte, die mit geringerer ausländischer Unterstützung operieren können, als sehr ehrgeizig. Von der geplanten Solderhöhung abgesehen, lassen die Maßnahmen der AfPak-Strategie nicht erkennen, wie die schwerwiegenden Strukturprobleme der afghanischen Armee beseitigt werden sollen: zu wenig geeignete Rekruten aufgrund von Analphabetismus und Mangelernährung, Patronage und Ämterkauf statt Leistungsauslese beim

25 Peter Baker und Thom Shanker, „In New Afghanistan Strategy, Obama will Add Troops", New York Times, 27.3.2009.

26 Vgl. Generalstabschef Admiral Michael Mullen, Abschrift des Pressegesprächs am 27.3.2009, S. 1, www.jcs.mil/chairman/speeches/0327officepressavail\%20. pdf.

27 Seth G. Jones, U.S. Strategy in Afghanistan, Stellungnahme vor dem Unterausschuss für den Mittleren Osten und Südasien des Auswärtigen Ausschusses des Repräsentantenhauses, Washington, D.C. / Santa Monica (RAND), 2.42009 , S. 5; www.rand.org/pubs/testimonies/CT324.

28 „Eine Zahl von 17.000 (...) im Laufe des Jahres 2009 mag politisch bequem sein, die Inanspruchnahme der Streitkräfte begrenzen und vermeiden, weitere Truppen aus dem Irak abziehen zu müssen. Aber es ist keineswegs klar, dass sie ausreicht, um Anforderungen gerecht zu werden, die Kommandeure im Feld auf 25.000 - 30.000 beziffern und andere Experten, darunter einige hochrangige US-Generale, eher bei 40.000 - 50.000 ansiedeln." Anthony H. Cordesman, The Afghan-Pakistan Conflict: US Strategic Options in Afghanistan, Washington, D.C. (CSIS), 20.3.2009, S. 18 (Arbeitsentwurf). (Übers., T.H.).

29 Damals wurden die Kosten hierfür auf jährlich fünf Milliarden US-Dollar in den ersten drei und circa drei Milliarden US-Dollar in den beiden folgenden Jahren geschätzt, vgl. Thom Shanker, „Pentagon backing plan to bolster Afghan Army“, International Herald Tribune, 9.-10.8.2008. Der Zielwert von 134.000 Mann umfasst 12.000 Mann in Ausbildung und Übergangsverwendungen, steht also für eine effektive Kampfstärke von 122.000, vgl. Obaid Younossi, Peter Dahl Thruelsen, Jonathan Vaccaro, Jerry M. Sollinger und Brian Grady, The Long March. Building an Afghan National Army, Santa Monica (RAND), 2009, S. xii.

30 Vgl. Antonio Giustozzi, „Auxiliary Force or National Army? Afghanistan's ,ANA' and the Counter-Insurgency Effort, 2002-2006“, in: Small Wars \& Insurgencies (Vol. 18, No. 1) March 2007, S. 47-67, Anthony H. Cordesman, David Kasten und Adam Mausner, Winning in Afghanistan: Creating Effective Afghan Security Forces, Washington, D.C. (CSIS), 6.1.2009 (Arbeitsentwurf) und C.J. Chivers, „Marching forward, falling back“, International Herald Tribune, 9.6.2009. 
Offizierskorps, ethnische Unausgewogenheiten und Probleme, verbreitetes unerlaubtes Fernbleiben von der Truppe, unzureichende Logistik, mangelnde Initiative und Kampfbereitschaft, Unfähigkeit zu Operationen oberhalb der Bataillons-Ebene, unzureichende und unzweckmäßige Ausstattung sowie fehlende Loyalität gegenüber der Zentralregierung.

\subsection{Realistische und messbare Ziele}

Die Fortschritte der AfPak-Strategie sollen an einem Kriterienkatalog gemessen werden. Kritiker haben der Regierung Bush entgegengehalten, sie könne weder ihre Fortschritte, noch ihre Rückschläge messen, da sie sich niemals auf quantifizierbare Ziele festgelegt habe. Das soll sich nun ändern. Die AfPakStrategie verfolge, so Holbrooke, „erreichbare Ziele mit ausrei- chenden Ressourcen, statt das Gegenteil“. ${ }^{31}$ Laut Riedel lägen die Kriterien und Maßstäbe noch nicht fest, würden aber zügig erarbeitet. $^{32}$

Die Riedel-Arbeitsgruppe orientiert sich am Beispiel der Truppenverstärkung im Irak (surge), die gleichfalls von der Einführung von Messgrößen für den militärischen Erfolg begleitet war. Hierauf hatte der Streitkräfteausschuss des Senats gedrängt. Sein Vorsitzender, Senator Carl Levin, lobte daher diesen Aspekt der AfPak-Strategie öffentlich. ${ }^{33}$ Insbesondere Vizepräsident Joseph R. Biden soll auf realistische Ziele und benchmarks

31 Holbrooke, Rede auf 45. Münchner Sicherheitskonferenz, a.a.O. (Fn. 15), S. 2.

32 Riedel, Press Briefing, a.a.o. (Fn. 5), S. 5. Man darf davon ausgehen, dass es sich überwiegend um die gleichen Messgrößen handeln wird, wie sie Anthony $\mathrm{H}$. Cordesman zugrunde legt, vgl. The Afghan-Pakistan War: The Rising Threat, 2002-2008, Washington, D.C. (CSIS), 27.1.2009.

33 Thom Shanker und Mark Mazetti, „U.S. to send thousands of trainers to Kabul“, International Herald Tribune, 27.03.2009.

Empfehlungen für die Politik der USA gegenüber Afghanistan und Pakistan

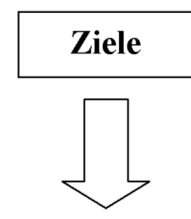

\begin{tabular}{|l|}
\hline - Zerstörung terroristischer Netzwerke in \\
Afghanistan und insbesondere Pakistan, \\
um die Vorbereitung und Durchführung \\
internationaler Terroranschläge zu \\
unterbinden \\
- Aufbau einer leistungsfähigen, legitimen \\
und durchsetzungsfähigen Regierung in \\
Afghanistan, die mit geringerer \\
auswärtiger Unterstützung \\
handlungsfähig ist \\
- Selbstständige afghanische \\
Sicherheitskräfte, welche die \\
Aufstandsbekämpfung mit verringerter \\
Unterstützung der USA anführen können \\
- Eine zivil kontrollierte, \\
verfassungsmäßige und stabile \\
Regierung in Pakistan und eine \\
Volkswirtschaft, die der pakistanischen \\
Bevölkerung Entwicklungschancen \\
eröffnet \\
- Einbeziehung der internationalen \\
Gemeinschaft in die Verfolgung dieser \\
Ziele für Afghanistan und Pakistan, mit \\
einer wichtigen Führungsrolle für die \\
Vereinten Nationen \\
\hline
\end{tabular}

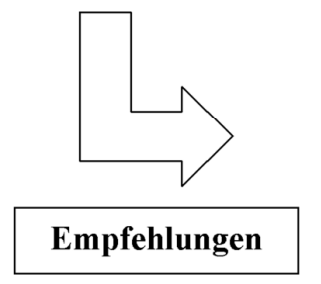

- In Afghanistan muss eine integrierte zivilmilitärische Strategie der Aufstandsbekämpfung verfolgt werden. Die erforderlichen Ressourcen sind bereitzustellen

- Der Aufbau der Zivilgesellschaft in Afghanistan genießt Vorrang und verlangt, dass ausreichende Mittel hierfür zur Verfügung gestellt werden

- Bis 2011 sind die Afghanische Nationalarmee auf eine Stärke von 134.000 und die Polizeikräfte auf eine Stärke von 82.000 zu bringen

- Die Integrität und Legitimität der Regierung Afghanistans sind zu stärken

- Die Regierung Afghanistans wird in ihrem Bemühen unterstützt, verständigungsbereite Aufständische zu integrieren

- Provinzregierungen und örtliche Verwaltungen werden in den zivilen Wiederaufbau einbezogen

- Der Bedingungszusammenhang zwischen Aufstandsbewegung und Drogenökonomie wird unterbrochen

- Für die Unterstützung unserer Ziele in Afghanistan wird größere internationale Unterstützung mobilisiert

- Die Zusammenarbeit zwischen Afghanistan und Pakistan wird gestärkt

- Die Regierung Pakistans wird stärker auf die Bekämpfung der gemeinsamen Bedrohung verpflichtet

- Pakistans Fähigkeiten, Extremisten zu bekämpfen, werden unterstützt und ausgebaut

- Die Wirtschaftshilfe für Pakistan wird erhöht und verbreitert

- Für die wirtschaftliche Zusammenarbeit mit Pakistan werden neue Bereiche erschlossen

- Die Regierung und Verwaltung Pakistans ist zu stärken, insbesondere in den Stammesgebieten unter Zentralverwaltung (FATA) und in der Provinz an der Nordwestgrenze

- Die Verbündeten werden um Hilfe für Afghanistan und Pakistan gebeten 
gedrängt haben, als unverzichtbare Argumentationshilfe, um in einer sehr schwierigen Wirtschaftslage den Kongress davon zu überzeugen, umfangreiche zusätzliche Mittel für die AfPakStrategie zu bewilligen. ${ }^{34}$

\section{Umsetzung}

\subsection{Politisch: Einwerbung von Unterstützung}

Nach Bekanntgabe der AfPak-Strategie machte sich die Regierung Obama unverzüglich an ihre Verwirklichung. Die internationale Afghanistan-Konferenz in Den Haag am 31. März 2009 und der NATO-Jubiläumsgipfel am 4. und 5. April 2009 in Straßburg und Kehl waren die ersten in schneller Folge getakteten Schritte, um die Zustimmung der Verbündeten und der internationalen Staatengemeinschaft zu gewinnen sowie konkrete Unterstützungsbeiträge einzuwerben.

Innenpolitisch bedarf die AfPak-Strategie der Unterstützung des US-Kongresses für zwei Gesetzespakete. Das erste umfasst die zusätzlichen Kosten für die US-Truppen im Irak und in Afghanistan für das laufende Jahr 2009 zusammen mit 400 Millionen US-Dollar Militärhilfe und 500 Millionen US-Dollar Wirtschaftshilfe für Pakistan sowie weiteren 980 Millionen US-Dollar Aufbauhilfe für Afghanistan. Mit dem zweiten Paket bittet die Regierung Obama um jeweils 1,5 Milliarden US-Dollar Hilfsgelder jährlich (für eine Laufzeit von fünf Jahren) für die Entwicklung der Zivilgesellschaft in Pakistan - insgesamt 7,5 Milliarden US-Dollar. Obwohl der Kongress der AfPak-Strategie der Regierung grundsätzlich wohlgesonnen scheint, ist die Zustimmung von Senat und Repräsentantenhaus nicht selbstverständlich. Beide Empfängerländer sind für Korruption und Fehlleitung von Hilfsgeldern berüchtigt. Pakistan war zudem in der Vergangenheit verantwortlich für die Weiterverbreitung von Kernwaffentechnik - kein Verhalten, das Volksvertreter in einer Wirtschaftskrise mit Steuergeldern belohnen wollen.

\subsection{Personalpolitisch: konsequente Erfolgs- orientierung}

Eine ganze Reihe von Personalmaßnahmen zeigt, dass die Regierung Obama die AfPak-Strategie entschlossen zum Erfolg führen will. Zunächst wurde der frühere Befehlshaber der US-Streitkräfte in Afghanistan, Generalleutnant a.D. Karl W. Eikenberry, zum neuen Botschafter in Kabul ernannt. Ihm wurde als Stellvertreter Francis J. Ricciardone zur Seite gestellt, vormals Botschafter in Ägypten und auf den Philippinen und ein Vertrauter Holbrookes. Mit der besseren Abstimmung der eigenen nichtmilitärischen Maßnahmen mit denen der Vereinten Nationen machte die Obama-Administration Ernst. Auf ihr Betreiben wurde der Berufsdiplomat Peter W. Galbraith zum Stellvertreter des norwegischen Leiters der VN-Mission in Afghanistan, Kai Eide, ernannt. Einem weiteren Diplomaten mit einschlägigen Erfahrungen, Timothy M. Carney, wurde die Lei-

34 Steven Erlanger und Helene Cooper, „President gains little amid NATO rifts“, International Herald Tribune, 6.4.2009. tung einer Arbeitsgruppe übertragen, die die Vorbereitung der Präsidentschaftswahlen im August 2009 unterstützt. Bis Juni will das US-Außenministerium 51 neue Stellen in Afghanistan besetzen. Bis zu 300 weitere Zivilbedienstete sollen zur Umsetzung der AfPak-Strategie nach Afghanistan entsandt werden. ${ }^{35}$ Doch es ist schwierig, die Stärkung des zivilen Wiederaufbaus personell zu unterlegen. Kurzfristig lässt sich das von Gates und anderen beklagte Ungleichgewicht zwischen uniformiertem und zivilem Personal im Dienste der US-Sicherheitspolitik nicht beheben, u.a. schlicht deshalb nicht, weil es zivilen Dienststellen an Rechtsgrundlagen fehlt, um Mitarbeiter ins Ausland versetzen zu können. So wurde das Pentagon aufgefordert, 300 Reservisten mit geeigneten Qualifikationsprofilen für den Aufbau der zivilen Infrastruktur zu benennen. Auch der Rückgriff auf private Personaldienstleister wird unvermeidlich sein. ${ }^{36}$

Die Personalie, die am meisten Aufmerksamkeit erregte, war die vorzeitige Ablösung des Oberkommandieren der US-Streitkräfte in Afghanistan, General David McKiernan, durch Generalleutnant Stanley A. McChrystal, der zuvor das Kommando Spezialkräfte (Joint Special Operations Command) befehligt hatte. Verteidigungsminister Gates und Generalstabschef Mullen hielten McKiernan keine konkreten Fehler vor. ${ }^{37}$ Sie wollten einen Oberkommandierenden haben, der sich unbelastet von der Vergangenheit 100-prozentig mit der neuen Strategie identifiziert. Alle sollen kraftvoll vom gleichen Notenblatt singen: eine bedingungslos erfolgsorientierte Personalpolitik.

\subsection{Pakistan: Militärische Eskalation}

McChrystal steht vor allem für die verstärkte Bekämpfung von Aufständischen auf pakistanischem Hoheitsgebiet. Auch wenn dies in der veröffentlichten Version der AfPak-Strategie nicht deutlich wird, so haben doch zwei Vorstudien den Einsatz von US-Spezialtruppen auch in der Provinz Belutschistan empfohlen. Deren Provinzhauptstadt Quetta gilt als Taliban- und Al-Qaida-Hochburg. McChrystal hatte seit Jahren auf den verstärkten Einsatz von Spezialtruppen in Pakistan gedrängt. Nun erfolgt er. ${ }^{38}$ Mehr als 70 Angehörige der Army Special Forces sind seit Sommer 2008 als Ausbilder, Berater und Spezialisten in den Aufstandsgebieten eingesetzt, wo sie eine 400-köpfige paramilitärische Kommandoeinheit der Grenztruppen (Frontier Corps) unterstützen. ${ }^{39}$ Selbstständige Kampfeinsätze haben US-Spezialeinheiten bisher nicht durchgeführt. Es darf angenommen

35 Karen De Young, „Civilians to Join Afghan Buildup“, Washington Post, 19.3.2009.

36 Thom Shanker, „Shorthanded, U.S. uses military to fill gap in Afghan rebuilding “, International Herald Tribune, 24.4.2009.

37 Auch der Luftangriff auf das afghanische Dorf Granai in der Provinz Fora, bei dem möglicherweise bis zu 117 Zivilisten getötet wurden, soll nicht der ausschlaggebende Anlass gewesen ein, obwohl die zivilen Opfer auch auf Fehlverhalten von US-Soldaten zurückzuführen sind, vgl. Carlotta Gall und Taimorr Shah, „Airstrikes brought horror, Afghan villagers say“, International Herald Tribune, 15.5.2009.; Eric Schmitt und Thom Shanker, „U.S. inquiry faults Afghan airstrikes", International Herald Tribune, 3.6.2009.

38 David E. Sanger und Eric Schmitt, „U.S. weighs expanding war in Pakistan“, International Herald Tribune, 18.3.2009; Eric Schmitt und Mark Mazetti, „,Order lets U.S. strike Al Qaeda worldwide“, International Herald Tribune, 11.11.2008.

39 Eric Schmitt und Jane Parlez, „U.S. trains Pakistanis in tribal regions“, International Herald Tribune, 23.2.2009. 
werden, dass die AfPak-Strategie hier künftig weitergehende Maßnahmen vorsieht.

Weil die Regierung Pakistans offiziell keine US-Truppen auf ihrem Staatsgebiet duldet, sind unbemannte Flugkörper (RPV, Remotely Piloted Verhicles) vom Typ Predator und Reaper die wichtigsten Mittel der USA zur Bekämpfung von Taliban und Al-Qaida in Pakistan. ${ }^{40}$ Bereits unter der Regierung Bush wurden die Abstimmungsverfahren festgelegt und die Zahl der Einsätze erhöht. Von Jahresbeginn bis Anfang Mai 2009 erfolgten 16 Angriffe, verglichen mit 36 im gesamten Jahr 2008. Nach Angaben hochrangiger US-Militärs wurden seit August 2008 mit RPV-Angriffen neun von 20 Taliban- und Al-Qaida-Kommandeuren getötet, die auf einer „Hit“-Liste der US-Geheimdienste standen. ${ }^{41}$

Militärs und CIA halten die RPV-Einsätze deshalb für erfolgreich. Kritiker verweisen hingegen auf die große Zahl getöteter Zivilisten. Bei einer Trefferquote von zwei Prozent könne man nicht von „Präzisionsangriffen“ sprechen. RPVs seien deshalb ungeeignet und sogar kontraproduktiv für eine nachhaltige Aufstandsbekämpfung. Da in Stammesgesellschaften wie jener der Paschtunen außerdem Tugenden wie Mut und Einsatzbereitschaft zählten, würden anonyme fliegende Roboter zu Symbolen für die fehlende Bereitschaft, sich tapfer für die Bevölkerung zu engagieren. ${ }^{42}$

Die sichtbar schwerwiegendste Folge der neuen AfPak-Strategie ist die Offensive der pakistanischen Sicherheitskräfte gegen die Taliban im Swat-Tal und in den Bezirken Dir und Buner. Sie wurde am 6. Mai 2009, pünktlich zum Dreier-Gipfeltreffen Obamas mit den Präsidenten Karsai und Zardari in Washington, eingeleitet. Inwieweit Grenztruppen und in welchem Umfang reguläre Armeeeinheiten eingesetzt sind, scheint noch unklar. Allerdings wurden mindestens 6.000 Soldaten von der Grenze zu Indien ins Kampfgebiet verlegt, wo sie bereits vor dem Terroranschlag in Mumbai stationiert waren. ${ }^{43}$ Gemessen an der Stärke der pakistanischen Streitkräfte von 500.000 ist das wenig, und eine Rückverlegung stellt keine zusätzliche Stärkung dar. Aber es ist ein erstes Zeichen dafür, dass das Drängen der USA, die Taliban als Bedrohung des pakistanischen Staates ernst zu nehmen, Wirkung zeigt.

Die menschlichen Opfer der Kampagne in Swat, Dir und Buner sind unübersehbar: Die Zahl der Flüchtlinge geht mindestens in die Hunderttausende. Sogar das Militär beziffert sie mit 1,3 Millionen. Andere Quellen sprechen von drei Millionen Flüchtlingen. Damit handelt es sich um die größte Flüchtlingsbewe-

40 Sie starten von einem Stützpunkt in Pakistan. Ihr Einsatz wird von der CIAZentrale in Langley, Virginia, gelenkt. Pakistanische Fluglotsen in der USBotschaft in Islamabad stellen sicher, dass die pakistanische Luftwaffe die RPVs und vorgeschobenen Beobachter, die vom Boden aus die Zielansprache koordinieren, nicht gefährden, vgl. Jane Parlez, „Pakstan protests, but backs drones", International Herald Tribune, 16.4.2009; Eric Schmitt und Christopher Drew, „U.S. to increase drone strikes on rebels“, International Herald Tribune, 9.4.2009.

41 Schmitt und Parlez, „U.S. trains ...“, a.a.O. (Fn. 39).

42 Marc Mazetti, „The Downside of Letting Robots Do the Bombing“, New York Times, 22.3.2009; David Kilcullen und Andrew McDonald Exum, „More harm than good“, International Herald Tribune, 19.5.2009.

43 Carlotta Gall und Elisabeth Bumiller, „Pakistan retakes key town from militants“, International Herald Tribune, 30.4.2009. gung in der Geschichte Pakistans seit der Trennung von Indien 1947. 44

\section{Kritische Beurteilung}

\subsection{Handwerkliche Schwächen aufgrund von Termindruck}

Die Arbeit an der AfPak-Strategie stand unter starkem Termindruck. Unterlassungen der Bush-Administration, die Ankündigungen Obamas im Wahlkampf, der Wunsch, die innenpolitische Aufbruchstimmung für außenpolitische Weichenstellungen zu nutzen, die afghanische Präsidentschaftswahl im August und der NATO-Gipfel Anfang April: All das zwang zu schnellen Ergebnissen. Angesichts dramatischer Lageeinschätzungen sonst besonnener Militärs („Abwärtsspirale“) ging es zuallererst darum, schnell irgendetwas vorzuschlagen, das geeignet erschien den Trend zu brechen. Trotz zahlreicher Vorarbeiten ist es eine respektable Leistung der Riedel-Arbeitsgruppe, innerhalb von 60 Tagen eine sicherheitspolitische Neuausrichtung herbeigeführt zu haben. Abstriche bei der Ergebnisqualität und handwerkliche Schwächen kann man der Arbeitsgruppe unter diesen Umständen kaum vorwerfen.

Zu den offensichtlichen Defiziten zählt die Vernachlässigung der Zwillingsprobleme Korruption und Drogenökonomie. Gerade weil sich die AfPak-Strategie zugute hält, sowohl die zivile als auch militärische Konfliktdimension sowie den afghanischen und den pakistanischen Kriegsschauplatz integriert zu betrachten, fällt auf, dass sie die Wechselwirkung zwischen Drogenökonomie und Gewalt und ihre Destabilisierung der Region vernachlässigt. Die wenigen Äußerungen zu den Alternativen für die Agrarwirtschaft bleiben auffallend oberflächlich und vage. ${ }^{45}$ Hochrangige Vertreter der Regierung Obama sprechen Korruption im afghanischen Regierungsapparat zwar ungewohnt deutlich an. Und die meisten Maßnahmen gegen dieses Problem erfordern Diskretion ${ }^{46}$, vor allem dann, wenn der zur Wiederwahl stehende Präsident mindestens ebenso sehr Teil des Problems wie Teil der Lösung zu sein scheint. Aber es fällt auf, dass Korruptionsbekämpfung in den lancierten Informationen und Interpretationen zur AfPak-Strategie keine Rolle spielt. Es verwundert, wie stark die AfPak-Strategie auf die Stärkung der Polizeikräfte setzt, wo doch das Innenministerium, dem diese unterstehen, als korruptestes Ressort in einer korrupten Regierung gilt. ${ }^{47}$

Der Zusammenhang zwischen Taliban und Drogenökonomie ist differenziert zu sehen. Weder haben die Taliban den Drogenanbau erfunden noch sind sie wirtschaftlich allein von ihm abhängig. Auch profitierten sie keineswegs allein von ihm. Die größten Gewinne werden nicht von den Produzenten, sondern

\footnotetext{
44 Salman Masood, „Easing Curfew lets Pakistan Refugees flee“, International Herald Tribune, 16.-17.5.2009.

45 Vgl. Press Briefing, a.a.O. (Fn. 5), S. 4. Holbrooke deutet als neue Idee an, Weizen könne eine Alternative zum Mohnanbau sein. US-Experten haben dies allerdings bereits vor Jahren geprüft und verworfen. Vgl. Ali A. Jalali, Robert B. Oakley und Zoe Hunter, „Combating Opium in Afghanistan“, in: Strategic Forum (Nr. 224), November 2006, S. 1-6, hier: S. 5.

46 Jones, U.S. Strategy in Afghanistan, a.a.O. (Fn. 27 ), S. $11 \mathrm{f}$.

47 Vgl. Cordesman et al, Winning in Afghanistan ..., a.a.O. (Fn. 30), S. 61, 64.
} 
auf den nachgelagerten Wertschöpfungsstufen erwirtschaftet. ${ }^{48}$ Wenn die US-Streitkräfte und ihre Verbündeten sich darauf beschränken, den Mohnanbau zu unterbinden, ohne den Bauern wirtschaftliche Alternativen anzubieten, werden sie alle relevanten Akteure der afghanischen Gesellschaft gegen sich aufbringen. Antworten auf diese Herausforderung bleibt die AfPak-Strategie schuldig.

\subsection{Militärische und zivile Mittel: ungleichge- wichtig und nicht integriert}

Die AfPak-Strategie hat den Anspruch, neben und zusätzlich zu militärischen Mitteln sehr viel stärker als bisher nichtmilitärische Instrumente einzusetzen. Im Katalog ihrer Ziele und Instrumente stehen militärische Mittel und zivilgesellschaftlicher Wiederaufbau jedoch eher unvermittelt nebeneinander. Die zivilen und militärischen Komponenten der AfPak-Strategie wirken ungleichgewichtig und nicht wirklich aufeinander abgestimmt. Dieser Strukturfehler ist erklärlich, gleichwohl aber schwerwiegend.

Die von Gates und anderen beklagte jahrelange Vernachlässigung nichtmilitärischer Mittel der US-Außen- und Sicherheitspolitik lässt sich nicht kurzfristig beheben. Es fehlt an zivilen US-Fachleuten in ausreichender Zahl und entsprechender Qualifikation (vgl. 3.2.). Es mangelt aber auch an einer gemeinsamen, integrierten Entwicklungsstrategie für die Zivilgesellschaften Afghanistans und Pakistans. In Afghanistan verfolgen die Vereinten Nationen, die ISAF-Regionalkommandos, ausländische Regierungsstellen und die zahlreichen Nichtregierungsorganisationen jeweils eigene Wiederaufbau- und Entwicklungsprogramme. Die US-Regierung hat ein Kompetenzund Glaubwürdigkeitsproblem, das es ihr unmöglich machen dürfte, diese Vielfalt zu integrieren - ein ohnehin ehrgeiziges Unterfangen. ${ }^{49}$ Die starke Hinwendung zum Leiter der VN-Mission in Afghanistan deutet darauf hin, dass die AfPak-Strategie die pragmatische Lösung dieses Problems darin sucht, durch und über die VN-Mission zu wirken.

Die unzureichende Integration militärischer und ziviler Mittel in der AfPak-Strategie wird aber auch am Problem ihrer Ungleichzeitigkeit deutlich. Während Präsident Obama noch vor Abschluss des AfPak-Strategy Review eine Truppenverstärkung in Afghanistan anordnen konnte, bedarf es für die Aufstockung der Wirtschaftshilfe für Afghanistan und Pakistan der Zustimmung des Kongresses. Dies wird einige Zeit in Anspruch nehmen. Die ersten neuen Gelder zur Stärkung der pakistanischen Zivilgesellschaft werden frühestens zum Herbst 2009 fließen können.

Sinnfällig wird die Ungleichzeitigkeit militärischer und ziviler Maßnahmen auch am Beispiel der Offensive der pakistanischen Sicherheitskräfte im Swat-Tal. Sie löste die größte Flüchtlingsbewegung aus, die Pakistan bisher gesehen hat. Ihre Bewälti-

48 Vgl. Sean M. Maloney, „On a pale horse? Conceptualizing narcotics production in southern Afghanistan and its relationship to the Narcoterror Nexus", in: Small Wars \& Insurgencies (Vol. 20, No. 1) March 2009, S. 203-214.

49 Natürlich kann von der AfPak-Strategie nicht erwartet werden, was bereits bei Abwesenheit von außen angetriebener kriegerischer Konflikte weder in Afghanistan noch in Pakistan gelungen ist: eine ausgewogene, sich selbst tragende wirtschaftliche und soziale Entwicklung. gung ist der Bevölkerung, internationalen Hilfsorganisationen und der überforderten pakistanischen Regierung überlassen. Vorbereitete Not- und Wirtschaftshilfe, die helfen würde das Flüchtlingselend zu mildern sowie die Herzen und Köpfe der Landbevölkerung zu gewinnen und gegen die pakistanischen Taliban einzunehmen, scheint nicht Bestandteil der AfPak-Strategie zu sein.

\subsection{Falsche Vorbilder, fehlerhafte Annahmen}

Interessanterweise wird der AfPak-Strategie gerade von ehemaligen Kommandeuren und ihren militärpolitischen Beratern entgegengehalten, sie orientiere sich am falschen Vorbild der Truppenverstärkung (surge) im Irak. Die Verhältnisse seien aber nicht vergleichbar. Wer im Irak die städtischen Verdichtungsräume kontrolliere, beherrsche das Land. Anders in Afghanistan, wo die Masse der Bevölkerung auf dem Land lebe und die Hauptstadt Kabul niemals beherrschenden Einfluss auf die Provinzen habe ausüben können. ${ }^{50}$

General Petraeus, dem als Oberkommandierenden des CENTCOM die US-Streitkräfte im Irak und in Afghanistan unterstehen und dem die geistige Vaterschaft für den Strategiewechsel im Irak zugesprochen wird, ${ }^{51}$ vermeidet sorgfältig den Eindruck, er würde das - angebliche - Erfolgsrezept für den irakischen Kriegsschauplatz umstandslos auf das AfPak-Kriegstheater anwenden. Sowohl wichtige Elemente der AfPak-Strategie als auch die Ernennung General McChrystals sehen Beobachter als untrügliches Zeichen dafür, dass Aufstandsbekämpfung (counterinsurgency, abgekürzt: COIN) das neue Paradigma für das Vorgehen der USA am Hindukusch geworden ist. Die COIN-Doktrin, so die Kritiker, impliziere die Legitimität der Regierung, die gegen illegitime Aufständische verteidigt werde. Diese Annahme träfe aber auf die Regierungen in Kabul und Islamabad nicht zu. Weite Teile Afghanistans seien der Regierung in Kabul gleichgültig - und umgekehrt: aufeinanderfolgende Regierungen in Islamabad und die sie tragenden Eliten hätten niemals für die nordwestlichen Grenzprovinzen Verantwortung übernommen. ${ }^{52}$ Kurzum: Counterinsurgency sei die falsche Doktrin zur Rettung scheiternder Staaten (failing states) mit massiven Funktions- und Legitimationsdefiziten. Sie tauge nicht als Ersatz für den Aufbau von Nationalstaatlichkeit (nation-building).

\subsection{Fehlende Nachhaltigkeit und fragliche Ausstiegstauglichkeit}

Der vorgesehene Aufbau von afghanischer Polizei und Afghanischer Nationalarmee erfordert viel Optimismus. Die Ausbildungshilfe der USA und der NATO-Verbündeten muss quantitativ und qualitativ deutlich verbessert werden. Es ist keineswegs sicher, ob die 4.000 Ausbilder, die von den USA zusätzlich ent-

$50 \mathrm{Vgl}$. Eric T. Olson, „Rethink the Afghan Surge. A US General explains why the Iraq model doesn't apply“, Christian Science Monitor, 17.3.2009; www.csmonitor.com/2009/0317/p09s01-coop.html. Der Autor befehligte zwischen 2004 und 2005 die NATO-Streitkräfte in Afghanistan.

51 Differenzierter hingegen Michael R. Gordon, „Months of administration debate preceded Iraq troop surge“, International Herald Tribune, 1.9.2008.

52 Celeste Ward, „Countering the Military's Latest Fad: Counterinsurgency“, Washington Post, 17.5.2009. Der Autor war 2006 Politischer Berater des USKommandeurs im Irak. 
sandt werden, und die 1.400 bis 2.000 Ausbilder, welche die Verbündeten auf dem NATO-Gipfel zugesagt haben, ${ }^{53}$ in zwei Jahren erreichen können, was in den vergangenen sechs Jahren nicht gelang. „Realistische Ziele“, auf die die AfPak-Strategie Wert legt, sehen anders aus. Die Frage, wer dauerhaft für den Sold der zusätzlichen Sicherheitskräfte aufkommt, ist ebenso klärungsbedürftig wie die nach der zusätzlichen Infrastruktur für ihre Unterbringung. Aus eigener Kraft wird der afghanische Staat den Aufbau der Sicherheitskräfte im angestrebten Umfang nicht finanzieren können. ${ }^{54}$

US-Sicherheitsfachleuten gilt die ANA als diejenige Regierungsinstitution, die in der afghanischen Bevölkerung das größte Ansehen genießt. Nicht zuletzt hat sie deshalb in der AfPakStrategie einen so hohen Stellenwert. Wenn aber sogar Erfolg beim Aufbau der ANA ein Zustand ist, den die Regierung Afghanistans aus eigenen Kräften nicht aufrechterhalten kann, dann stellt sich die Frage, ob die AfPak-Strategie überhaupt vernünftig und nachhaltig ist und als Einstieg in den Ausstieg von USA und NATO aus Afghanistan taugt.

\subsection{In den Zielen unklar}

Am Rande des NATO-Gipfels erklärten hochrangige Diplomaten, dass es allen Verbündeten natürlich um eine Ausstiegsstrategie (exit strategy) gehe. ${ }^{55}$ Das veröffentlichte AfPakStrategiepapier nennt als Ziele legitime und handlungsfähige Regierungen in beiden Ländern, betont also nation-building. Für General Petraeus ist hingegen „unser grundlegendes Ziel in Afghanistan, dass transnationale Terrorristen nicht wieder geschützte Rückzugsräume errichten, wie vor dem 11. September. "56 In Vorstudien wurden diese unterschiedlichen Ziele - denial und control - offenbar noch analytisch sauber getrennt. In der Praxis bleibt die Frage offen: Geht es „lediglich“ darum sicherzustellen, dass die Gebiete am Hindukusch nicht wieder zum Vorbereitungsraum für Terrorangriffe werden? Oder sollen mit Hilfe der Aufstandsbekämpfung in Afghanistan und Pakistan um ein staatliches Gewaltmonopol herum Staatsstrukturen nach westlichem Vorbild errichtet werden ${ }^{57}$

Bruce Riedel zog aus seiner Analyse 2007 folgenden Schluss: „Al-Qaida [...] benötigt gescheiterte Staaten, um sich zu entwickeln". ${ }^{58}$ Ist damit der Umkehrschluss richtig, dass nur der erfolgreiche Export des westlichen Staatsmodells wirksam vor fundamentalistischem Terror schützt? Eine solche „Strategie“

53 Erlanger und Cooper, „President gains little ...“, a.a.O. (Fn. 34).

54 Die Denkfabrik RAND gelangt zu folgendem Ergebnis: „Selbst wenn die Afghanische Nationalarmee (ANA) das Stadium erreicht, in dem sie selbständig operieren kann, so werden die Vereinigten Staaten und andere Nationen noch für eine beträchtliche Zeit eine Sicherheitspräsenz im Lande beibehalten müssen. Darüber hinaus wird wahrscheinlich auf absehbare Zeit ein internationales Engagement erforderlich bleiben, um die ANA und ihre Infrastruktur aufrechtzuerhalten." Younossi et al, The Long March, a.a.O. (Fn. 29), S. xiv, (Übers., T.H.).

55 Erlanger und Cooper, „President gains little ...“, a.a.O. (Fn. 34).

56 David H. Petraeus, „The Future of the Alliance and the Mission in Afghanistan“, Rede auf der 45. Münchner Sicherheitskonferenz am 8.2.2009; www. securityconference.de/konferenzen/rede/php?menu_2009=\&menu_konfere nzen $=\&$ sprache $=$ de\&id $=264 \&$.

57 Sanger und Schmitt, „U.S. weighs ..“, a.a.O. (Fn. 38).

58 Riedel, „Al Quade Strikes Back ...“, a.a.O. (Fn. 4), S. 37. würde den Westen schlicht überfordern und kann nicht erfolgreich sein. ${ }^{59}$

Die vorgelegte AfPak-Strategie „löst“ den Zielkonflikt, wie es für bürokratische Politik typisch ist: kumulativ. Das heißt beide Ziele werden gleichzeitig nebeneinander verfolgt. Die Rangfolge und das wechselseitige Verhältnis der Ziele nation-building und denial bleiben in der AfPak-Strategie letztlich ungeklärt. Das Ziel einer Beendigung der Intervention (exit) wird nicht offen thematisiert. Hierin liegt die konzeptionelle Schwäche von AfPak. Als eine Strategie mit zwei konkurrierenden Zielen hat sie geringe Erfolgsaussichten. Indem sie den Ausstieg an das ehrgeizige Ziel eines erfolgreichen nation-building zu knüpfen scheint, überfordert sie zumindest die NATO-Verbündeten, aber möglicherweise auch die USA selbst.

\section{Beurteilung: unklare Ziele, nicht integrierte Mittel - fraglicher Erfolg}

Angesichts der sich verschlechternden Sicherheitslage am Hindukusch wurde vielfach ein Strategiewechsel verlangt, auch in dieser Zeitschrift. ${ }^{60}$ Die AfPak-Strategie führt ihn nun herbei. Sie umfasst alle geforderten Elemente und Instrumente - und geht sogar noch darüber hinaus. AfPak ist im Ansatz pragmatisch, fortschrittlich und stellt eine deutliche Verbesserung gegenüber der (Nicht-)Strategie der Regierung Bush dar. Dennoch hat sie erhebliche Schwächen, Risiken und Nebenwirkungen. Der militärische Ressourceneinsatz wirkt maßvoll. Unterziele sind teilweise ungerechtfertigt optimistisch. Militärische und zivile Instrumente sind unausgewogen und nicht wirklich integriert. Insbesondere die Bekämpfung der Korruption und Alternativen zur Drogenökonomie werden vernachlässigt. AfPak thematisiert zwar die Probleme am Hindukusch zutreffend. Doch die Lösungsansätze sind demgegenüber weniger überzeugend. Eine militärische Eskalation in Pakistan erscheint militärisch kurzsichtig und politisch höchst gefährlich. Der konzeptionelle Fehler der AfPak-Strategie besteht darin, dass sie sich zwischen dem „realistischen“ Ziel Terrorbekämpfung (denial) und dem „idealistischen“ Ziel Entwicklung (nation-building) nicht entscheidet. Damit zeigt sie auch keine Ausstiegsperspektive (exit) auf. Ohne diese werden aber Legitimation, Bündnisfähigkeit, Nachhaltigkeit und Erfolgsaussichten der AfPak-Strategie fraglich. Eine wirklichkeitstaugliche Ausstiegsperspektive ist aber notwendig, zumal die Ressourcen für die Intervention am Hindukusch nicht unbegrenzt zur Verfügung stehen. Insbesondere eines ist in der Öffentlichkeit der westlichen Demokratien knapp: Zeit. Die aber haben andere reichlich. Wie Talibanführer Mullah Omar 2001 feststellte: „Die Amerikaner haben alle Uhren. Aber wir haben alle Zeit." ${ }^{1}$

59 Vgl. zur Strategiekritik im „Krieg gegen den Teror“: Patrick Porten, „Long wars and long telegrams: containing Al-Qaeda“, in: International Affairs (Vol. 85, No. 2) March 2009, S.285-305.

60 Hans-Georg Ehrhart und Roland Kaestner, „Das internationale Engagement in Afghanistan: Plädoyer für einen Strategiewechsel“, in: Sicherheit und Frieden (27. Jg., Nr. 1) 2009, S. 7-13.

61 Zit. nach: Richard deVillafranca, „Reconsidering Afghanistan: Time for an ,Azimuth Check'“, in: Parameters (Vol. 38, No. 4) Winter 2008/2009, S. 77-94, hier: S. 77; (Übers., T.H.). 